\title{
Parametrising the attractor of the two-dimensional Navier-Stokes equations with a finite number of nodal values
}

\author{
Peter K. Friz ${ }^{\mathrm{a}, 1}$, James C. Robinson ${ }^{\mathrm{b}, *, 2}$ \\ ${ }^{\text {a }}$ Trinity College, University of Cambridge, Cambridge CB2 1TQ, UK \\ ${ }^{\mathrm{b}}$ Mathematics Institute, University of Warwick, Coventry CV4 7AL, UK
}

Received 20 December 1999; received in revised form 4 September 2000; accepted 20 September 2000

Communicated by R. Temam

\begin{abstract}
We consider the solutions lying on the global attractor of the two-dimensional Navier-Stokes equations with periodic boundary conditions and analytic forcing. We show that in this case the value of a solution at a finite number of nodes determines elements of the attractor uniquely, proving a conjecture due to Foias and Temam. Our results also hold for the complex Ginzburg-Landau equation, the Kuramoto-Sivashinsky equation, and reaction-diffusion equations with analytic nonlinearities. (C) 2001 Elsevier Science B.V. All rights reserved.
\end{abstract}

Keywords: Global attractor; Determining nodes; Navier-Stokes equations; Gevrey regularity

\section{Introduction}

Foias and Temam showed in [21] that a sufficient number of "nodal values" will determine the asymptotic behaviour of a solution of the 2D incompressible Navier-Stokes equations. In particular, they showed that if $u(x, t)$ and $v(x, t)$ are two solutions of the equation

$$
\frac{\partial u}{\partial t}-v \Delta u+(u \cdot \nabla u)+\nabla p=f, \quad \nabla \cdot u=0, \quad x \in \Omega
$$

with either Dirichlet or periodic boundary conditions, then there exists a distance $\delta$ such that for any finite collection of nodes in $\Omega,\left\{x_{1}, \ldots, x_{k}\right\}$ with

$$
\min _{1 \leq j \leq k}\left|x-x_{j}\right| \leq \delta \quad \text { for all } x \in \Omega,
$$

then if

$$
\sup _{1 \leq j \leq k}\left|u\left(x_{j}, t\right)-v\left(x_{j}, t\right)\right| \rightarrow 0 \quad \text { as } t \rightarrow \infty,
$$

\footnotetext{
* Corresponding author.

E-mail address: jcr@maths.warwick.ac.uk (J.C. Robinson).

${ }^{1}$ Present address: Courant Institute of Mathematical Sciences, New York University, 251 Mercer Street, New York, NY 10012-1185, USA.

${ }^{2}$ JCR is a Royal Society University Research Fellow.
} 
one has

$$
\sup _{x \in \Omega}|u(x, t)-v(x, t)| \rightarrow 0 \quad \text { as } t \rightarrow \infty .
$$

Since the asymptotic behaviour on this collection of nodes fixes the asymptotic behaviour of the whole solution, they have been termed a set of "determining nodes". Since then, better estimates of the maximum separation $\delta$ which guarantees this result have been obtained by Foias et al. [19], Foias and Titi [23] and Jones and Titi [35,36] (see also [3] for a more general discussion of "determining functionals").

What this result does not say is that the values of $u(x, t)$ at these nodes determine instantaneously the values of $u(x, t)$ throughout the domain. In general, we cannot expect this to be the case, since there is no reason to believe that the transient behaviour is determined by a finite number of degrees of freedom. However, if we restrict to the long-time asymptotic behaviour it seems more plausible, and it was conjectured by Foias and Temam in their paper that the nodal values uniquely determine the elements of the global attractor.

This global attractor $\mathcal{A}$ describes all possible long-term configurations of the system. More precisely, if the solutions of a PDE define a dynamical system on a Banach space $B$, so that

$$
u\left(t ; u_{0}\right)=S(t) u_{0}
$$

with the solution operator $S(t)$ satisfying the semigroup properties

$$
S(0)=\mathrm{id}, \quad S(t+s)=S(t) S(s), \quad \lim _{t \rightarrow 0} S(t) u_{0}=u_{0},
$$

then the global attractor is a compact subset of $B$ which is invariant, i.e.

$$
S(t) \mathcal{A}=\mathcal{A} \text { for all } t \geq 0,
$$

and attracting, i.e.

$$
\operatorname{dist}_{B}(S(t) X, \mathcal{A}) \rightarrow 0 \quad \text { as } t \rightarrow \infty
$$

for any bounded set $X \subset B$. In (4), dist ${ }_{B}$ is the Hausdorff semi-distance in $B$, i.e.

$$
\operatorname{dist}_{B}(X, Y)=\sup _{x \in X} \inf _{y \in Y}\|X-Y\|_{B} .
$$

See [31] or [57] for more details.

In this paper, we show that the conjecture of Foias and Temam is true, for the case of periodic boundary conditions with a forcing function which lies in an analytic Gevrey class.

In fact we prove a result valid under general assumptions, and then show that these hold for the 2D Navier-Stokes equations, the complex Ginzburg-Landau equation (1D and 2D), the scalar Kuramoto-Sivashinsky equation, and reaction-diffusion equations in any dimension. Precisely, for $\tau>0$ we let $\mathcal{G}_{\tau}$ denote the Gevrey class of functions (see Section 2 for full details)

$$
\mathcal{G}_{\tau}=D\left(\mathrm{e}^{\tau A^{1 / 2}}\right)
$$

where $A$ denotes the negative Laplacian operator, and we prove the following theorem.

Theorem 1. Let $\Omega$ be a periodic domain in $\mathbb{R}^{n}$, and let $\mathcal{A}$ be a finite dimensional compact subset of $\mathbb{L}^{2}(\Omega)=$ $\left[L^{2}(\Omega)\right]^{m}$, which in addition is uniformly bounded in $G_{\tau}$. Then provided that $k>16 n d_{f}(\mathcal{A})$, for almost every set of $k$ nodes

$$
\mathbf{x}=\left(x_{1}, \ldots, x_{k}\right), \quad x_{j} \in \Omega,
$$


the values of $u\left(x_{j}\right)$ uniquely determine the function $u \in \mathcal{A}$. ["Almost every" is with respect to nk-dimensional Lebesgue measure.]

We say that almost every set of $k$ nodes are instantaneously determining; we show in Section 7 that such a set of nodes are also "determining" in the sense of Foias and Temam.

That the attractor is bounded in a Gevrey class is used in two important ways. First, along with the finite-dimensionality of the attractor it allows us to obtain a parametrisation of the attractor which is Hölder continuous in the parameters, with the Hölder exponent as close to 1 as we wish. Secondly, that the functions are analytic allows us to control the structure of their zero sets, which is fundamental to our approach. Indeed, observe that without the assumption of analyticity such a result is not possible under similar hypotheses. For a simple example take a one-parameter family of $C^{\infty}$ functions on $[-1,1]$

$$
u(x ; \epsilon)= \begin{cases}0, & x \leq 0, \\ \mathrm{e}^{-\epsilon / x^{2}}, & x>0\end{cases}
$$

with $\epsilon \in[1,2]$, and observe that no number of nodes in $[-1,0]$ will suffice to determine which value of $\epsilon$ we have chosen.

A similar result to Theorem 1 could be obtained on replacing $G_{\tau}$ by the space $D\left(A^{r} \mathrm{e}^{\tau A^{s}}\right)$ for any $r \geq 0$ and any $s>0$. For simplicity, we restrict below to the case $r=0$ and $s=\frac{1}{2}$ which arises naturally in the examples that we have consider in Section 6.

We note that this is not the first result which gives a parametrisation of the attractor using a finite number of variables. Indeed, as long ago as 1981 Mañé [45] proved an abstract result along these lines, and we use a strengthened version of his result due to Hunt and Kaloshin [33] in our proof. However, to our knowledge this is the first result which provides an experimentally realisable method of parametrisation.

\section{Gevrey classes of analytic functions}

The main assumption in the proof is that the global attractor is a bounded finite-dimensional subset of a suitable Gevrey class of functions. We restrict to the case of periodic boundary conditions (see comments at the end of this section), and for simplicity consider a domain $\Omega=[0,2 \pi]^{n}-$ of course, similar results apply when $\Omega=\left[0, L_{1}\right] \times \cdots \times\left[0, L_{n}\right]$.

The negative Laplacian on $\Omega, A=-\Delta$, is a linear self-adjoint unbounded non-negative operator on $L^{2}(\Omega)$, and has a complete set of orthogonal eigenfunctions, just the Fourier modes $\mathrm{e}^{\mathrm{i} j \cdot x}$ for all $j \in \mathbb{Z}^{n}$, which satisfy

$$
A \mathrm{e}^{\mathrm{i} j \cdot x}=|j|^{2} \mathrm{e}^{\mathrm{i} j \cdot x} .
$$

The space $\mathbb{L}^{2}(\Omega)=\left[L^{2}(\Omega)\right]^{m}$ can be identified (cf. [59]) with the space of all functions $u$ satisfying

$$
u=\sum_{j \in \mathbb{Z}^{n}} u_{j} \mathrm{e}^{\mathrm{i} j \cdot x}, \quad u_{j} \in \mathbb{C}^{m}, \quad u_{-j}=\bar{u}_{j}
$$

with

$$
(2 \pi)^{n} \sum_{j \in \mathbb{Z}^{n}}\left|u_{j}\right|^{2}=|u|_{\mathbb{L}^{2}}^{2}=\sum_{j=1}^{m}\left|u_{j}\right|_{L^{2}}^{2}<\infty .
$$


We can also consider $A$ as an operator on $\mathbb{L}^{2}(\Omega)$, so that for $u \in \mathbb{L}^{2}, A$ acts on each component of $u$. It follows that the domain of $A, D(A)$, and of any of its positive powers, $D\left(A^{k}\right)$, can be characterised as those $u$ of the form (5) for which

$$
(2 \pi)^{n} \sum_{j}|j|^{4 k}\left|u_{j}\right|^{2}=\left|A^{k} u\right|^{2}<\infty
$$

(where $|\cdot|$ is the $\mathbb{L}^{2}$ norm).

We will use multi-index notation, so that $\alpha=\left(\alpha_{1}, \ldots, \alpha_{n}\right)$, and

$$
\partial^{\alpha}=\partial_{1}^{\alpha_{1}} \ldots \partial_{n}^{\alpha_{n}} .
$$

We also write $|\alpha|=\alpha_{1}+\cdots+\alpha_{n}, \alpha !=\alpha_{1} ! \ldots \alpha_{n} !$, and $x^{\alpha}=x_{1}^{\alpha_{1}} \ldots x_{n}^{\alpha_{n}}$. We will need some inequalities concerning factorial functions; these all follow from the identity

$$
\left(x_{1}+\cdots+x_{n}\right)^{k}=\sum_{|\alpha|=k} \frac{k !}{\alpha !} x^{\alpha},
$$

setting $x_{1}=\cdots=x_{n}=1$, we obtain

$$
\sum_{|\alpha|=k} \frac{k !}{\alpha !}=n^{k}
$$

and setting $n=2$ gives

$$
2^{-2 k}(2 k) ! \leq(k !)^{2} \quad \text { and } \quad \frac{(k+r) !}{k !} \leq 2^{k+r} r !
$$

For $u$ of the form (5), we have formally

$$
\partial^{\alpha} u=\sum_{j} i^{|\alpha|} j^{\alpha} u_{j} \mathrm{e}^{\mathrm{i} j \cdot x},
$$

so that

$$
\left|\partial^{\alpha} u\right|^{2}=(2 \pi)^{n} \sum_{j}\left|j^{\alpha}\right|^{2}\left|u_{j}\right|^{2} .
$$

Since

$$
\|u\|_{H^{k}}^{2}=\sum_{|\alpha| \leq k}\left|\partial^{\alpha} u\right|^{2},
$$

we consider $s=\left(j_{1}^{2}, \ldots, j_{n}^{2}\right)$, so that

$$
\sum_{|\alpha| \leq k}\left|j^{\alpha}\right|^{2}=\sum_{|\alpha| \leq k} s^{\alpha} .
$$

Now, using (6), we have

$$
\sum_{|\alpha|=k} s^{\alpha} \leq \sum_{|\alpha|=k} \frac{k !}{\alpha !} s^{\alpha}=|s|^{k},
$$


and $|s| \leq|j|^{2}$, hence it follows that

$$
\sum_{|\alpha| \leq k}\left|j^{\alpha}\right|^{2} \leq \sum_{l=0}^{k}|j|^{2 l} \leq 2|j|^{k},
$$

which yields

$$
\|u\|_{H^{k}}^{2} \leq 2(2 \pi)^{n} \sum_{j}|j|^{2 k}\left|u_{j}\right|^{2}=2\left|A^{k / 2} u\right|^{2} .
$$

For $\tau>0$, the Gevrey class $G_{\tau}$ is defined to be $D\left(\mathrm{e}^{\tau A^{1 / 2}}\right)$, the domain in $\mathbb{L}^{2}(\Omega)$ of the operator $\mathrm{e}^{\tau A^{1 / 2}}$, i.e. all those $u$ of the form (5) with

$$
(2 \pi)^{n} \sum_{j} \mathrm{e}^{2 \tau|j|}\left|u_{j}\right|^{2}=\left|\mathrm{e}^{\tau A^{1 / 2}} u\right|^{2}<\infty .
$$

Following [22], we will write the norm on $G_{\tau}$ as

$$
|u|_{\tau}=\left|\mathrm{e}^{\tau A^{1 / 2}} u\right| .
$$

In the proof of the main theorem, we assume that the functions in $\mathcal{A}$ are uniformly bounded in $G_{\tau}$ for some $\tau$. Fundamental to all that follows will be the following lemma.

Lemma 2. Suppose that $\Omega=[0,2 \pi]^{n}$, and that $u \in G_{\tau}$ as defined above. Then $u$ is real analytic and can be extended to an analytic function on the region

$$
S=\left\{z: \operatorname{Re} z \in \Omega,|\operatorname{Im} z| \leq \frac{\tau}{4 n}\right\}
$$

with

$$
\sup _{z \in S}|u(z)| \leq \beta|u|_{\tau},
$$

where $\beta$ is a constant depending only on $n$ and $\tau$.

Proof. If $u=\sum_{j} u_{j} \mathrm{e}^{\mathrm{i} j \cdot x}$ as in (5), then from (9)

$$
\begin{aligned}
|u|_{\tau}^{2} & =\left|\mathrm{e}^{\tau A^{1 / 2}} u\right|^{2}=(2 \pi)^{n} \sum_{j} \mathrm{e}^{2 \tau|j|}\left|u_{j}\right|^{2}=(2 \pi)^{n} \sum_{j, k} \frac{(2 \tau|j|)^{k}}{k !}\left|u_{j}\right|^{2} \\
& =(2 \pi)^{n} \sum_{k} \frac{(2 \tau)^{k}}{k !} \sum_{j}|j|^{k}\left|u_{j}\right|^{2}=\sum_{k} \frac{(2 \tau)^{k}}{k !}\left|A^{k / 4} u\right|^{2} .
\end{aligned}
$$

It follows that

$$
\left|A^{k / 4} u\right|^{2} \leq k !(2 \tau)^{-k}|u|_{\tau}^{2} \text { for all } k,
$$

from which

$$
\left|A^{k / 2} u\right|^{2} \leq \tau^{-2 k} 2^{-2 k}(2 k) !|u|_{\tau}^{2} .
$$


Now, $2^{-2 k}(2 k) ! \leq(k !)^{2}$ using $(7)$, and so

$$
\left|A^{k / 2} u\right| \leq \tau^{-k} k !|u|_{\tau}
$$

(We have shown, unsurprisingly, that $D\left(\mathrm{e}^{\tau A^{1 / 2}}\right) \subset D\left(A^{k}\right)$ for all $k$.) Using the standard Sobolev embedding result (e.g. [58])

$$
C^{k}(\Omega) \subset H^{k+(n / 2)+1}(\Omega),
$$

and (8), we obtain

$$
\left\|\partial^{\alpha} u\right\|_{\infty} \leq C^{\prime}\left|A^{(k+(n / 2)+1) / 2} u\right| \leq C^{\prime}\left(\frac{1}{\tau}\right)^{k+1+(n / 2)}\left(k+1+\frac{n}{2}\right) !|u|_{\tau}
$$

where $C^{\prime}$ is uniform over $k$. Now, using (7) this estimate becomes

$$
\left\|\partial^{\alpha} u\right\|_{\infty} \leq\left[C^{\prime}\left(\frac{2}{\tau}\right)^{1+(n / 2)}\left(\frac{1+n}{2}\right) !\right]\left(\frac{2}{\tau}\right)^{k} k !|u|_{\tau} .
$$

We write this as

$$
\left\|\partial^{\alpha} u\right\|_{\infty} \leq C|u|_{\tau}\left(\frac{2}{\tau}\right)^{k} k !
$$

where $k=|\alpha|$ and $C(\tau, n)=C^{\prime}(2 / \tau)^{1+(n / 2)}(1+n / 2)$ !

Now (following [54, Theorem 19.9]; cf. [34, Section 3], [10] and also [41]) write $u$ as the Taylor sum (cf. [53, Exercise 9.30])

$$
u(x)=\sum_{|\alpha| \leq k} \frac{\partial^{\alpha} u(a)}{\alpha !}(x-a)^{\alpha}+\sum_{|\alpha|=k+1} \frac{\partial^{\alpha} u(a+(x-a) t)}{\alpha !}(x-a)^{\alpha}
$$

for some $t \in(0,1)$. Since, we can estimate the final term (over $|\alpha|=k+1)$ using the bound in (12) as

$$
\left|\sum_{|\alpha|=k+1} \frac{\partial^{\alpha} u(a+t(x-a))}{\alpha !}(x-a)^{\alpha}\right| \leq C|u|_{\tau} \sum_{|\alpha|=k+1} \frac{(2 / \tau)^{k+1}(k+1) !}{\alpha !}|x-a|^{k+1}=C|u|_{\tau}\left(\frac{2 n}{\tau}\right)^{k+1}|x-a|^{k+1},
$$

using (6). Taking $|x-a| \leq \delta<\tau / 2 n$ the final term tends to zero as $k \rightarrow \infty$, from which it follows that $u(x)$ can be written as the Taylor series

$$
u(x)=\sum_{|\alpha| \geq 0} \frac{\partial^{\alpha} u(a)}{\alpha !}(x-a)^{\alpha}
$$

for all real $x$ with $|x-a| \leq \delta<\tau / 2 n$. Since the series converges absolutely, it is in fact convergent for all $x$ (complex values too) with $|x-a| \leq \delta$. The function $u(x)$ is therefore real analytic, and can be extended to an analytic function for all $z$ with $|z-x| \leq \delta$ for some $x \in \Omega$.

For (11) in the statement of the lemma we now use the Taylor expansion, (6) and (12) as above to obtain

$$
\begin{aligned}
\|u\|_{\infty} & \leq C|u|_{\tau}\left\{\sum_{|\alpha| \geq 0} \frac{(2 / \tau)^{|\alpha|}|\alpha| !}{\alpha !}|x-a|^{|\alpha|}\right\}=C|u|_{\tau} \sum_{k=0}^{\infty}\left(\frac{2 n}{\tau}\right)^{k}|x-a|^{k} \leq C|u|_{\tau} \sum_{k=0}^{\infty}\left(\frac{2 n \delta}{\tau}\right)^{k} \\
& \leq \frac{C}{1-(2 n \delta / \tau)}|u|_{\tau},
\end{aligned}
$$


provided that $|x-a| \leq \delta<\tau / 2 n$. If we take $\delta=\tau / 4 n$, we get

$$
\sup _{|\operatorname{Im} z| \leq \delta}|u(z)| \leq 2 C|u|_{\tau}
$$

which is (11) with $\beta=2 C$.

The use of periodic boundary conditions significantly simplifies the problem, since it is only in this case that one can expect, in general, to obtain a function which has the same radius of analyticity throughout the whole domain $\Omega$. John [34, Section 7, 1(b)] shows that for the equation $u_{t}-\Delta u=0$ on a domain $\Omega$ with smooth boundary, if the boundary conditions are Dirichlet $\left(\left.u\right|_{\partial \Omega}=0\right)$ then the radius of analyticity of a solution $u(x, t)$ at a point $x \in \Omega$ can only be bounded below by $\operatorname{dist}(x, \partial \Omega)$, which shrinks to zero as one approaches the boundary. Grujić and Kukavica [28] obtain a similar result for the scalar nonlinear heat equation $u_{t}=\Delta u+u^{k}$.

We treat the case of Dirichlet boundary conditions in a separate paper [25].

\section{Parametrising finite-dimensional sets}

We will need to use two different measures of the dimension of arbitrary sets. The Hausdorff dimension will prove useful since it is stable under countable unions,

$$
d_{H}\left(\bigcup_{j=1}^{\infty} X_{j}\right) \leq \max _{j} d_{H}\left(X_{j}\right)
$$

whereas the fractal dimension $d_{f}$ has the property that if $d_{f}(X)<\infty$ then $X$ can be embedded "nicely" in a finite-dimensional Euclidean space.

We first discuss the weaker of the two notions, the Hausdorff dimension

\subsection{Hausdorff dimension}

The Hausdorff dimension is based on the definition of the $d$-dimensional Hausdorff measure, which is essentially a generalisation of Lebesgue measure to "fractional dimensions". Indeed, later on we will use the fact that for integer values of $d, d$-dimensional Hausdorff and Lebesgue measure are proportional [15, Theorem 1.12].

To find the $d$-dimensional Hausdorff measure of a set $X$, take a cover of $X$ by balls $B\left(x_{i}, r_{i}\right)$ with radii $r_{i} \leq \epsilon$, and define

$$
\mu(X, d, \epsilon)=\inf \left\{\sum_{i} r_{i}^{d}: r_{i} \leq \epsilon \text { and } X \subseteq \cup_{i} B\left(x_{i}, r_{i}\right)\right\} .
$$

The $d$-dimensional Hausdorff measure of $X, \mathcal{H}^{d}(X)$, is given by

$$
\mathcal{H}^{d}(X)=\lim _{\epsilon \rightarrow 0} \mu(X, d, \epsilon)
$$

and the Hausdorff dimension of $X, d_{H}(X)$ is essentially that value of $d$ (if any) for which $\mathcal{H}^{d}(X)$ takes a finite value

$$
d_{H}(X)=\inf _{d>0}\left\{d: \mathcal{H}^{d}(X)=0\right\}
$$


We will need the stability of $d_{H}$ under countable unions (which is (13)), and the fact that if $f: X \rightarrow Y$ is a Hölder continuous function, so that

$$
\left\|f\left(x_{1}\right)-f\left(x_{2}\right)\right\|_{Y} \leq C\left\|x_{1}-x_{2}\right\|_{X}^{\theta}, \quad 0<\theta \leq 1,
$$

then if $E \subset X$

$$
d_{H}(f(E)) \leq \frac{d_{H}(E)}{\theta} .
$$

For proofs of these facts see [16].

A stronger definition of dimension allows us to parametrise "finite dimensional" sets using a finite number of parameters.

\subsection{The "fractal" dimension}

The "fractal" dimension of $X, d_{f}(X)$, is based on a covering of $X$ by a collection of balls of fixed radius. In finite-dimensional spaces the following definition is the same as that of the "upper box-counting dimension"; however, in infinite-dimensional spaces we are forced to consider balls rather than boxes since the "Hilbert cube" (consisting of $u=\sum_{j=1}^{\infty} c_{j} e_{j}$ with $\left\{e_{j}\right\}$ a countable basis of $H$ and $\left|c_{j}\right| \leq 1$ ) contains elements with arbitrarily large norm. We therefore define the fractal dimension of $X$ as

$$
d_{f}(X)=\lim \sup _{\epsilon \rightarrow 0} \frac{\log N(X, \epsilon)}{\log (1 / \epsilon)},
$$

where $N(X, \epsilon)$ is the minimum number of balls of radius $\epsilon$ necessary to cover $X$.

That an embedding theorem holds for sets with finite fractal dimension but not necessarily for those with finite Hausdorff dimension is a consequence of the inequality

$$
d_{f}(X \times Y) \leq d_{f}(X)+d_{f}(Y)
$$

a similar expression does not hold for the Hausdorff dimension. We note that the fractal dimension also obeys (14). (Ref. [14] contains a nice discussion of the difference between the Hausdorff and fractal dimensions.)

\subsection{An embedding theorem}

We will use the following theorem, due to Hunt and Kaloshin [33], which guarantees that there are many linear maps from a Banach space $B$ into $\mathbb{R}^{k}$ (for some $k$ ) which are embeddings of a finite-dimensional set. In particular, their result gives a bound on the Hölder constant of the parametrisation of $X$ which results from this embedding. (Mañé [45] first showed the existence of such embeddings, with Foias and Olson [20] first guaranteeing the Hölder property of the inverse.)

In the statement of the theorem, the "thickness" of $X, \tau(X)$, is given by

$$
\tau(X)=\lim \sup _{\epsilon \rightarrow 0} \frac{\log d(X, \epsilon)}{\log (1 / \epsilon)}
$$

where $d(X, \epsilon)$ is the minimum dimension of all finite-dimensional subspaces, $V$, of $B$ such that every point of $X$ lies within $\epsilon$ of $V$. 
Theorem 3 (Hunt and Kaloshin [33]). If $X$ is a compact subset of a Banach space B then, provided that D is an integer with $D>2 d_{f}(X)$ and

$$
\theta<\frac{D-2 d_{f}(X)}{D(1+\tau(X))}
$$

a dense set of linear maps from $B$ into $\mathbb{R}^{D}$ have the following properties:

1. they are injective on $X$,

2. their inverse is Hölder continuous from $L X$ into $X$ with exponent $\theta$.

(We note here that their result is in fact slightly stronger, giving a "prevalent" set of linear maps with this Hölder property, see $[32,61]$.)

It was shown in [24] that when the set $X$ consists of infinitely differentiable functions then $\tau(X)=0$; however, the result in that paper considers the case when $X$ is a subset of $L^{2}$, and we will find it convenient to consider $X$ as a finite-dimensional subset of the Gevrey class $G_{\varphi \tau}$ for some $0<\varphi<1$. The following lemma shows that if $X$ is bounded in $G_{\tau}$ and has finite fractal dimension in $\mathbb{L}^{2}(\Omega)$, then in fact it is a zero-thickness finite-dimensional subset of $G_{\varphi \tau}$ for all $0 \leq \varphi<1$.

Lemma 4. Suppose that $X$ is a bounded subset of $G_{\tau}$. Then, for $0 \leq \varphi<1$, the thickness of $X$ measured in $G_{\varphi \tau}$, is zero. Furthermore, if the fractal dimension of $X$ in $\mathbb{L}^{2}, d_{f}\left(X, \mathbb{L}^{2}\right)$, is finite, the fractal dimension in $G_{\varphi \tau}$, $d_{f}\left(X, G_{\varphi \tau}\right)$, is bounded as

$$
d_{f}\left(X, G_{\varphi \tau}\right) \leq \frac{d_{f}\left(X, \mathbb{L}^{2}\right)}{1-\varphi} .
$$

Proof. It is convenient to denote by $w_{j}$ and $\lambda_{j}$ the eigenfunctions and eigenvalues of $A=-\Delta$, ordered so that $\lambda_{j+1} \geq \lambda_{j}$. Since $\Omega$ is a periodic domain in $\mathbb{R}^{n}$, the eigenvalues of $A$ are proportional to sums of $n$ square integers, and so in particular $\lambda_{j^{n}} \sim j^{2}$. It follows that $\lambda_{j} \sim j^{2 / n}$.

Now, if $u=\sum_{i} c_{i} w_{i}$ we let $P_{j}$ denote the projection on to the first $j$ eigenfunctions, and $Q_{j}=I-P_{j}$. Then

$$
\begin{aligned}
\left|u-P_{j} u\right|_{\varphi \tau} & =\left|Q_{j} u\right|_{\varphi \tau}=\left|\mathrm{e}^{-(1-\varphi) \tau A^{1 / 2}} \mathrm{e}^{(1-\varphi) \tau A^{1 / 2}} Q_{j} u\right|_{\varphi \tau} \leq\left\|\mathrm{e}^{-(1-\varphi) \tau A^{1 / 2}} Q_{j}\right\|_{\text {op }}\left|\mathrm{e}^{(1-\varphi) \tau A^{1 / 2}} u\right|_{\varphi \tau} \\
& =\left\|\mathrm{e}^{-(1-\varphi) \tau A^{1 / 2}} Q_{j}\right\|_{\text {op }}|u|_{\tau} \leq \mathrm{e}^{-(1-\varphi) \tau \lambda_{j+1}^{1 / 2}|u|_{\tau} .}
\end{aligned}
$$

Since $\lambda_{j} \sim j^{2 / n}$ it follows that (for some $c>0$ )

$$
\left|u-P_{j} u\right|_{\varphi \tau} \leq \mathrm{e}^{-c \tau j^{1 / n}}|u|_{\tau},
$$

which implies that, for some $C>0$,

$$
d(X, \epsilon) \leq C(-\log \epsilon)^{n} .
$$

It follows that $\tau(X)=0$ in $G_{\varphi \tau}$.

To prove (16), we show that the identity map on the attractor is a Hölder map from $\mathbb{L}^{2}$ into $G_{\varphi \tau}$ with exponent $1-\varphi$, and then use (14). For $u, v \in X$, set $w=u-v$ and write

$$
w=\sum_{j} c_{j} w_{j} .
$$


Then, using Hölder's inequality

$$
|w|_{\varphi \tau}^{2}=\sum_{j} \mathrm{e}^{2 \varphi \tau \lambda_{j}^{1 / 2}}\left|c_{j}\right|^{2} \leq\left(\sum_{j} \mathrm{e}^{2 \tau \lambda_{j}^{1 / 2}}\left|c_{j}\right|^{2}\right)^{\varphi}\left(\sum_{j}\left|c_{j}\right|^{2}\right)^{1-\varphi},
$$

so that

$$
|w|_{\varphi \tau} \leq|w|_{\tau}^{\varphi}|w|^{1-\varphi} .
$$

Since $X$ is bounded in $G_{\tau}$, the Hölder property follows, and we can deduce (16) using (14).

We will use a corollary, a combination of Lemma 4 and Theorem 3, in the proof of the main theorem.

Corollary 5. Under the hypotheses of Lemma 4, for each $D>2 d_{f}\left(X, \mathbb{L}^{2}\right)$ and each

$$
\theta<1-\frac{2 d_{f}\left(X, \mathbb{L}^{2}\right)}{D}
$$

there exists a $\varphi_{0}>0$ such that for each $0 \leq \varphi<\varphi_{0}$ there is a parametrisation of $X$ using D parameters which is Hölder continuous into $G_{\varphi \tau}$, with Hölder exponent $\theta$.

Proof. Choose $\varphi_{0}$ small enough that

$$
D>\frac{2 d_{f}\left(X, \mathbb{L}^{2}\right)}{\left(1-\varphi_{0}\right)}
$$

and

$$
\theta<1-\frac{2 d_{f}\left(X, \mathbb{L}^{2}\right)}{\left(1-\varphi_{0}\right) D}
$$

The result then follows from Lemma 4 and Theorem 3.

\section{Zero sets of analytic functions}

We will need the following Hölder implicit function theorem to investigate the structure of the zero sets of functions $u(x ; \underline{\epsilon})$ analytic in $x$ and Hölder continuous in $\underline{\epsilon}$.

Theorem 6. Let $E \subset \mathbb{R}^{D}$, and let $u(x ; \underline{\epsilon})$ be a function from $\mathbb{R} \times E$ into $\mathbb{R}$ which is $C^{1}$ in $x$, Hölder continuous in $\underline{\epsilon}$ with exponent $\theta$, i.e.

$$
\left|u\left(x ; \underline{\epsilon}_{1}\right)-u\left(x ; \underline{\epsilon}_{2}\right)\right| \leq C\left|\underline{\epsilon}_{1}-\underline{\epsilon}_{2}\right|^{\theta},
$$

and with $u_{x}(x ; \underline{\epsilon})$ continuous in $\underline{\epsilon}$. Then if

$$
u\left(x_{0} ; \underline{\epsilon}_{0}\right)=0 \quad \text { and } \quad u_{x}\left(x_{0} ; \underline{\epsilon}_{0}\right) \neq 0
$$

there exists a neighbourhood of $\left(x_{0}, \underline{\epsilon}_{0}\right)$ in $\mathbb{R} \times E$, and a Hölder continuous function $x(\underline{\epsilon})$ with exponent $\theta$, such that

$$
u(x(\underline{\epsilon}), \underline{\epsilon})=0
$$

and $x(\underline{\epsilon})$ is the unique zero of $u(x, \underline{\epsilon})$ in this neighbourhood. 
Proof. For a proof of the result in this form, without the usual assumption of differentiability in $\epsilon$, one can follow the proof in Hale [30, Chapter 0, Theorem 3.3] which uses the uniform contraction mapping theorem. The Hölder continuity of $x(\underline{\epsilon})$ in $\underline{\epsilon}$ follows from the Hölder continuity of $u(x ; \underline{\epsilon})$ in $\underline{\epsilon}$, once again employing an argument from Hale [30, Chapter 0, Theorem 3.2].

We now apply this to generalise a lemma from a paper of Yamazato [60] concerning the zero sets of real analytic functions. We call a set a Hölder $n$-manifold if it is given locally as the image of $\mathbb{R}^{n}$ under a Hölder continuous function $f$. Note that it follows from (14) that the Hausdorff or fractal dimension of this manifold can only be bounded by $n / \theta$, where $\theta$ is the Hölder exponent of $f$.

Proposition 7. Let $E \subset \mathbb{R}^{D}$, and suppose that $u\left(x_{1}, \ldots, x_{n} ; \underline{\epsilon}\right)$ is real analytic in $x \in \mathbb{R}^{n}$ and Hölder continuous in $\underline{\epsilon} \in E$ together with all its partial derivatives in $x$ (all with exponent $\theta$ ). In addition we suppose the function $u(., \underline{\epsilon})$ is not identically zero for any $\underline{\epsilon} \in E$. Then the zero set of $u(x ; \underline{\epsilon})$, viewed as a subset of $\mathbb{R}^{n} \times E \subset \mathbb{R}^{n} \times \mathbb{R}^{D}$, is contained within a countable collection of Hölder $D+n-1$-manifolds, given in the form

$$
\left(x^{\prime}, x_{j}\left(x^{\prime}, \underline{\epsilon}\right) ; \underline{\epsilon}\right)
$$

where $x^{\prime}=\left(x_{1}, \ldots, x_{j-1}, x_{j+1}, \ldots, x_{n}\right)$, and $x_{j}$ is a Hölder function of its arguments with exponent $\theta$.

In the proof we write $e_{i}$ for the multi-index $\left(\delta_{i 1}, \ldots, \delta_{i n}\right)$, where $\delta_{i j}$ is the Kronecker delta.

Proof. We assume that $u$ is a function into $\mathbb{R}$, since if $u$ takes values in $\mathbb{R}^{m}$ with $m>1$, the zero set of $u$ is a subset of the zero set of any of the components of $u$.

We cannot apply the implicit function Theorem 6 (IFT) to every point in the zero set $Z=u^{-1}(0 ; 0)$, since there may be points at which derivatives of $u$ are zero. The idea is to apply the IFT repeatedly, removing manifolds of zeros of decreasingly high order derivatives of $u$. Finally, we end up with a set on which the IFT applies to gives the set of all remaining zeros. Each set we consider is a Hölder $D+n-1$ manifold.

We will assume that $Z$ is non-empty, otherwise we are finished. So take $\left(x^{0} ; \underline{\epsilon}^{0}\right) \in Z ; u\left(., \underline{\epsilon}^{0}\right)$ is analytic and not identically 0 . Thus there exists a multi-index $\alpha=\left(\alpha_{1}, \ldots, \alpha_{n}\right)$ (depending on $\underline{\epsilon}$ ) such that $\partial^{\alpha} u\left(x^{0}, \underline{\epsilon}^{0}\right) \neq 0$ and $\partial^{\beta} u\left(x^{0}, \underline{\epsilon}^{0}\right)=0$ for all multi-indices $\beta$ s.t. $\beta_{j} \leq \alpha_{j}, j=1, \ldots, n$, and $\beta \neq \alpha$. (If not then the analyticity of $u$ would imply that $u \equiv 0$.) As $\alpha$ is not $(0, \ldots, 0)$, there exists an integer $i_{1}$ with $\alpha_{i_{1}} \geq 1$. For simplicity of presentation, we assume that $\alpha_{1} \geq 1$.

By the preceding IFT, we get an open neighbourhood $U_{0}=U\left(x^{0} ; \underline{\epsilon}^{0}\right)$ of $\left(x^{0} ; \underline{\epsilon}^{0}\right)$ such that $W_{0}=U_{0} \cap\left[\partial^{\alpha-e_{1}}\right.$ $u=0]$ is represented as

$$
(x ; \underline{\epsilon})=\left(g_{0}\left(x^{\prime}, \underline{\epsilon}\right), x_{2}, \ldots, x_{n} ; \underline{\epsilon}\right),
$$

where $g_{0}$ is a Hölder continuous function and $x^{\prime}, \underline{\epsilon}$ vary in a neighbourhood of $\left(x_{2}^{0}, \ldots, x_{n}^{0} ; \underline{\epsilon}^{0}\right)$. We take $W_{0}$ as the first Hölder manifold in our collection. Note that, by the definition of $\alpha,\left(x^{0} ; \underline{\epsilon}^{0}\right)$ is contained in $W_{0}$. It suffices to show that all zeros of $u$ contained in $U^{0}$ can be represented as stated in the formulation of the theorem. In the case that $\alpha-e_{1}=(0, \ldots, 0)$ we have finished; otherwise there exists a second integer $i_{2}$ with $\left(\alpha-e_{1}\right)_{i_{2}} \geq 1$. As before, for simplicity of presentation we will treat the particular case $i_{2}=2$ (if $i_{2}=i_{1}$ then the argument would be similar). By removing $W_{0}$ we have excluded all points from $U^{0}$ where we cannot apply the IFT again; indeed, on $U_{0} \backslash W_{0}$ we have $\partial^{\alpha-e_{1}} u \neq 0$.

We now consider $W_{1}:=\left(U_{0} \backslash W_{0}\right) \cap\left[\partial^{\alpha-e_{1}-e_{2}} u=0\right]$. For any $\left(x^{1} ; \underline{\epsilon}^{1}\right) \in W_{1}$ the assumptions for the IFT are fulfilled, and we get open neighbourhoods of $\left(x^{1} ; \underline{\epsilon}^{1}\right)$, say $U_{1}$, w.l.o.g. contained in $U_{0} \backslash W_{0}$, where $\left[\partial^{\alpha-e_{1}-e_{2}} u=0\right]$ is given as $(x ; \underline{\epsilon})=\left(x_{1}, g_{1}\left(x^{\prime}, \underline{\epsilon}\right), x_{3}, \ldots, x_{n} ; \underline{\epsilon}\right), g_{1}$ being a Hölder function. The (a priori uncountable) union over 
all $\left(x^{1} ; \underline{\epsilon}^{1}\right) \in W_{1}$ of all these open neighbourhoods is an open set in $U_{0} \backslash W_{0}$ covering $W_{1}$. Lindelöf's Theorem (see, e.g. Kuratowski [42, Chapter II, Section 17]) allows us to reduce this to a countable union. $W_{1}$ is therefore covered by a countable union of open sets, and in each of them $W_{1}$ is represented as a Hölder manifold. So $W_{1}$ is contained in a countable union of such manifolds. If $\alpha-e_{1}-e_{2}=(0, \ldots, 0)$ we have finished. Otherwise we continue as before now considering the set $\left(U_{0} \backslash W_{0}\right) \backslash W_{1}$ where we can apply the IFT again.

This algorithm stops after $\sum \alpha_{i}$, a finite number of steps. Note that in the last step $W_{\text {last }}$ really consists of zeros of $u$. All the $W_{i}$ s before were thrown away for "security reasons".

\section{Proof of main theorem}

In this section, we use the results of Sections 3 and 4 to prove the main theorem of this paper. We now restate Theorem 1 in a slightly different, but equivalent formulation, and then give the proof.

Theorem 8. Let $\Omega$ be a periodic domain in $\mathbb{R}^{n}$, and $\mathcal{A}$ a finite-dimensional compact subset of $\mathbb{L}^{2}(\Omega)=\left[L^{2}(\Omega)\right]^{m}$ which is bounded in $G_{\tau}$ for some $\tau>0$. Then, provided that $k>16 n d_{f}(\mathcal{A})$, the map from $\mathcal{A}$ into $\mathbb{R}^{m k}$ given by

$$
E_{\mathbf{x}}: u \mapsto\left(u\left(x_{1}\right), \ldots, u\left(x_{k}\right)\right)
$$

is 1-1 between $\mathcal{A}$ and its image for almost every $\mathbf{x}=\left(x_{1}, \ldots, x_{k}\right)$ in $\Omega^{k}$ (with respect to $n k$-dimensional Lebesgue measure).

Proof. We investigate the set of nodal values $\mathbf{x}$ for which the map $E_{\mathbf{x}}$ fails to be 1-1. For such an $\mathbf{x}$ there must exist two functions in $\mathcal{A}, u$ and $v$, such that

$$
u\left(x_{j}\right)=v\left(x_{j}\right) \text { for all } 1 \leq j \leq k .
$$

Equivalently, if we define

$$
X=\{w: w=u-v, u, v \in \mathcal{A}\},
$$

then there must exist a non-zero function $w \in X$ such that

$$
w\left(x_{j}\right)=0 \text { for all } 1 \leq j \leq k .
$$

Since we want to exclude zero, we define

$$
X^{0}=X \backslash\{0\} .
$$

Note that $d_{f}\left(X^{0}\right) \leq d_{f}(X) \leq 2 d_{f}(\mathcal{A})$, since $X$ is the image of $\mathcal{A} \times \mathcal{A}$ (with $d_{f}(\mathcal{A} \times \mathcal{A}) \leq 2 d_{f}(\mathcal{A})$ using (15)) under the Lipschitz map $(u, v) \mapsto u-v$ (use (14)).

We study the zeros of functions in $X^{0}$ and the collections of $k$ such zeros. We show that the union of all such collections over all functions in $X^{0}$ has Hausdorff dimension less than $n k$ if $k$ is sufficiently large. This will imply that the collection of "bad" nodes has measure zero.

The first step is to use Theorem 3 to find a linear map into $\mathbb{R}^{D}$, with $D>4 d_{f}(\mathcal{A})$, such that a parametrisation of $X^{0}$ is given by the $D$ parameters $\underline{\epsilon} \in \mathbb{R}^{D}$. We use the fact that $X$ is bounded in $G_{\tau}$ to deduce from Corollary 3.3 that for some $0<\varphi<1$ we can obtain a parametrisation of $X^{0}, w(x ; \epsilon)$, which is Hölder continuous from $\mathbb{R}^{D}$ into $G_{\varphi \tau}$, with Hölder exponent anything less than $1-\left(4 d_{f}(\mathcal{A}) / D\right)$. 
It follows from (11) that the parametrisation is also continuous into the space of continuous functions on the "strip" $S$ defined in Lemma 2 by (10), replacing $\tau$ by $\varphi \tau$. It then follows from the Cauchy integral formula (see, e.g. Range [51, Theorem 1.3])

$$
w(x ; \underline{\epsilon})=(2 \pi \mathrm{i})^{-n} \int_{C} \frac{w(\zeta ; \underline{\epsilon})}{\left(\zeta_{1}-z_{1}\right) \ldots\left(\zeta_{n}-z_{n}\right)} \mathrm{d} \zeta_{1} \ldots \mathrm{d} \zeta_{n},
$$

(where $C=\left\{\zeta \in \mathbb{C}^{n}:\left|\zeta_{i}-z_{i}\right|=\delta\right\}$, with $\delta$ such that all contours lie in the domain of analyticity of $w$ ) that all the derivatives of $w(x ; \underline{\epsilon})$ also depend in a Hölder way on $\underline{\epsilon}$, with the same exponent $\theta$.

We are thus led to consider a parametrised family of functions

$$
w(x ; \underline{\epsilon}), \quad x \in \Omega, \quad \underline{\epsilon} \in E,
$$

where $E=L X^{0}$ is the parameter space, a bounded subset of $\mathbb{R}^{D}$, and $w(x ; \underline{\epsilon})$ and all its derivatives depend in a Hölder continuous way on $\underline{\epsilon}$.

Now, Proposition 7 guarantees that the zero set of the function $w(x ; \underline{\epsilon})$ in $\Omega \times E$ is contained in a countable number of manifolds which are given in the form

$$
\left(x^{\prime}, x_{j}\left(x^{\prime}, \underline{\epsilon}\right) ; \underline{\epsilon}\right)
$$

where $x^{\prime}=\left(x_{1}, \ldots, x_{j-1}, x_{j}, \ldots, x_{n}\right)$, and $x_{j}$ is a Hölder function of its arguments. Observe that there are $D+(n-1)$ parameters; $D$ of these are always $\underline{\epsilon}$.

Now consider the set which consists of collections of $k$ zeros of $w(x ; \underline{\epsilon})$, as a subset of $\Omega^{k} \times E$, i.e. each element is

$$
\left(x^{1}, \ldots, x^{k}\right) \times\{\underline{\epsilon}\}, \quad x^{j} \in Z(w(\cdot ; \underline{\epsilon})),
$$

where $Z(w(\cdot ; \underline{\epsilon}))$ is the zero set in $\mathbb{R}^{n}$ of the function $w(x ; \underline{\epsilon})$ for fixed $\underline{\epsilon}$.

This set is clearly a countable union of Hölder $m$-manifolds, with $m$ no more than $k[D+(n-1)]$. We show in fact that each manifold is a $D+k(n-1)$-manifold. Indeed, the $x^{1}$ component of the product is given in the form

$$
\left(x^{1^{\prime}}, x_{j_{1}}^{1}\left(x^{1^{\prime}}, \underline{\epsilon}\right) ; \underline{\epsilon}\right)
$$

and in general the $x^{i}$ component is given as

$$
\left(x^{i^{\prime}}, x_{j_{i}}^{i}\left(x^{j^{\prime}}, \underline{\epsilon}\right) ; \underline{\epsilon}\right) .
$$

In other words, the $D$ parameters $\underline{\epsilon}$ are common to all components, and otherwise there are an additional $k(n-1)$ parameters. Since the set is given as a Hölder function of these parameters, it lies within a Hölder $D+k(n-1)$ manifold.

It follows that the Hausdorff dimension of each manifold is bounded by

$$
\frac{D+k(n-1)}{\theta},
$$

since it is the image of a subset of $\mathbb{R}^{D+k(n-1)}$ under a Hölder map with exponent $\theta$ (using (14)). Since $\theta$ can be taken arbitrarily close to $1-\left(4 d_{f}(\mathcal{A}) / D\right)$, this can be made as close as required to

$$
\frac{D+k(n-1)}{1-\left(4 d_{f}(\mathcal{A}) / D\right)} \text {. }
$$

Since the Hausdorff dimension is stable under countable unions (13), the Hausdorff dimension of the set of all such zeros, a subset of the union of all these countable manifolds, is bounded by the same quantity. Since projections are 
Lipschitz, it follows that this quantity also bounds the Hausdorff dimension of the projection of this set onto $\Omega^{k}$ (using (14)). This is precisely the collection of all sets of $k$ zeros over all functions in $X^{0}$

To ensure that this set has dimension less than $n k$, we need

$$
k>\frac{D^{2}}{D-4 n d_{f}(\mathcal{A})},
$$

the value of $D$ which gives the smallest value of $k$ is $D=8 n d_{f}(\mathcal{A})$, which gives $k>16 n d_{f}(X)$.

It follows from the definition of Hausdorff dimension (see Section 3.1) that the set of all "bad" choices of nodes has $n k$-dimensional Hausdorff measure zero. Since $n k$-dimensional Hausdorff measure and $n k$-dimensional Lebesgue measure are proportional (see [15, Theorem 1.12]) it follows that this set has Lebesgue measure zero.

Thus almost every choice of $k$ nodes is instantaneously determining.

\section{Applications}

We now show that our theorem applies to several examples.

\subsection{The 2D Navier-Stokes equations}

We first show that our theorem applies to the 2D Navier-Stokes equations,

$$
u_{t}-v \Delta u+(u \cdot \nabla) u+\nabla p=f, \quad \nabla \cdot u=0
$$

with periodic boundary conditions and a Gevrey regular forcing function. For simplicity we follow the standard presentation, in which we assume that $f$ and $u_{0}$ both have zero average over $\Omega$ (cf. [12,22,59], see also [8,57]).

The existence of a global attractor, with finite fractal dimension in $\mathbb{L}^{2}(\Omega)$, is shown by Constantin et al. [9] (see also [12]); the best estimate of this dimension is of the order of

$$
G^{2 / 3}(1+\log G)^{1 / 3},
$$

where $G$ is the Grashof number, $G=L^{2}|f| / v^{2}$. The Gevrey regularity of solutions was considered by Foias and Temam [22], who showed that if $f \in D\left(\mathrm{e}^{\sigma A^{1 / 2}}\right)$ for some $\sigma>0$ then the attractor is uniformly bounded in $D\left(A^{1 / 2} \mathrm{e}^{\tau A^{1 / 2}}\right)$, for some $\tau>0$. By considering the Fourier representation (cf. (9))

$$
A^{1 / 2} \mathrm{e}^{\tau A^{1 / 2}} u=(2 \pi)^{2} \sum_{j \in \mathbb{Z}^{2}}|j| \mathrm{e}^{2 \tau|j|}\left|u_{j}\right|^{2}
$$

it is clear that the attractor is also bounded in $G_{\tau}$.

It follows that our theorem applies in this case, and that the number of nodes necessary is bounded by $32 d_{f}(\mathcal{A})$, so of the order $G^{2 / 3}(1+\log G)^{1 / 3}$.

We will compare this to the classical, heuristic, length scale estimates due to [37] (see also [58]; Doering and Gibbon [12] give a much more detailed discussion of such length scales, and Eden et al. [13] give a very good summary of the various bounds). Kraichnan's theory constructs a length scale from the viscous enstrophy dissipation and the forcing. The enstrophy dissipation $\chi$ is given by

$$
\chi=v\langle\Delta u\rangle^{2} \equiv \frac{v}{L^{2}} \lim \sup _{t \rightarrow \infty} \sup _{u_{0} \in \mathcal{A}} \frac{1}{t} \int_{0}^{t}|A u(s)|^{2} \mathrm{~d} s,
$$


and a standard bound in the analysis of the 2D NSE with periodic boundary conditions (see, e.g. [59])

$$
v \int_{0}^{T}|A u(s)|^{2} \mathrm{~d} s \leq \frac{T}{v}|f|^{2}+\left\|u_{0}\right\|^{2},
$$

gives

$$
\chi \leq \frac{|f|^{2}}{L^{2} v}=v^{3} L^{-6} G^{2} .
$$

The only length that can be formed from $\chi$ and $v$ is

$$
L_{\chi}=\left(\frac{v^{3}}{\chi}\right)^{1 / 6}
$$

which yields

$$
\left(\frac{L_{\chi}}{L}\right) \sim G^{-1 / 3} \text {. }
$$

If we space our nodes evenly over the domain, then the separation required by our theorem is of the order of $G^{-1 / 3}$, with logarithmic corrections, confirming the Kraichnan length scale by analytically rigorous means. Note also that this is an entirely natural way to produce a length-scale from the equations, and ties in with the heuristic argument that one would expect that

$$
d_{f}(\mathcal{A}) \sim\left(\frac{L}{L_{\chi}}\right)^{2} .
$$

(See, e.g. [12])

The same argument applies to the 3D equations, provided that one assumes regularity (in the sense of Constantin et al. [7]), although in this case the estimate of the dimension of the attractor and hence of the number of nodes is much larger (see [7,58] or [26]).

For more discussion of this case see Ref. [52].

\subsection{The complex Ginzburg-Landau equation}

Gevrey regularity for the complex Ginzburg-Landau equation

$$
u_{t}-(1+\mathrm{i} v) \Delta u+(1+\mathrm{i} \mu)|u|^{2} u-a u=0
$$

on a periodic domain in dimensions 1 and 2 was shown by Promislow [50], and by Doelman and Titi [10] (also in those cases in which one can prove existence and uniqueness of solutions in 3D); Kukavica [39] gives a proof of the Gevrey regularity in the $1 \mathrm{D}$ case only.

Doering et al. [11] and Bartucelli et al. [1] show that the equation has a finite-dimensional global attractor, and the above results then guarantee that this attractor is bounded in some Gevrey class $G_{\rho}$ (for an appropriate $\rho>0$ ).

It follows that our theorem holds for the complex Ginzburg-Landau equation in dimensions 1 and 2, and in 3 dimensions in those cases for which one can prove existence and uniqueness (see [10]).

\subsection{The Kuramoto-Sivashinsky equation}

Collet et al. show [4] that the 1D Kuramoto-Sivashinsky equation

$$
u_{t}+u_{x x x x}+u_{x x}+u u_{x}=0, \quad u(x, t)=u(x+L, t),
$$


possesses a finite-dimensional global attractor in $L^{2}(0, L)$, and [5] that this attractor is bounded in the Gevrey class $G_{\rho}$ for some $\rho>0$. (For the restricted case of odd initial conditions such results were obtained previously — the existence of an attractor by Nicolaenko et al. [48] and the Gevrey regularity by Liu [43]; some further details on Gevrey regularity for solutions on the attractor are given by Grujić [29].) It follows that our results hold in this case also.

\subsection{Reaction-diffusion equations with analytic nonlinearity}

For scalar reaction-diffusion equations

$$
\frac{\partial u}{\partial t}-v \Delta u=f(u),
$$

on periodic domains in $\mathbb{R}^{n}$ the existence of finite-dimensional attractors is shown in Marion [46] and Temam [48], provided that $f$ is $C^{2}$ and satisfies the estimates

$$
-k-\alpha|s|^{p} \leq f(s) s \leq k-\beta|s|^{p} \quad \text { and } \quad f^{\prime}(s) \leq l
$$

for all $s \in \mathbb{R}$. The Gevrey regularity of solutions has been shown for polynomial nonlinearities by Promislow [50], and for general analytic nonlinearities with a majorising function (see below) by Ferrari and Titi [17] (reaction-diffusion equations on the two-dimensional sphere are treated in a similar way in [2]). The main assumption from [17] is that if

$$
f(u)=\sum_{j=0}^{\infty} a_{j} u^{j},
$$

then the majorising function

$$
g(s)=\sum_{j=0}^{\infty}\left|a_{j}\right| s^{j}
$$

converges for all $s \in \mathbb{R}$. Under this condition they show that if the attractor is bounded in the Sobolev space $H_{\text {per }}^{p}$ for $p>\frac{1}{2} n$ then it is also bounded in the Gevrey class $D\left(A^{p / 2} \mathrm{e}^{\rho A^{1 / 2}}\right)$. Since Marion [47, Theorem 5.2] shows that in this case the attractor is in fact bounded in $H_{\text {per }}^{k}(\Omega)$ for all $k$, the required Gevrey regularity follows and our theorem applies to reaction-diffusion equations in all space dimensions.

\subsection{Gevrey regularity in other examples}

We note that other equations are amenable to the Gevrey analysis, although the existence of finite-dimensional attractors is unresolved: the weakly damped driven nonlinear Schrödinger equation is treated by Oliver and Titi [49]; Bénard convection in a porous medium by Ly and Titi [44], and the Navier-Stokes equation on the whole of $\mathbb{R}^{2}$ by Grujić and Kukavica [27]. For use of Gevrey regularity to analyse other problems see [39-41].

\section{Discussion}

\subsection{Instantaneously determining nodes are also asymptotically determining}

A simple corollary of our result shows that instantaneously determining nodes are also "determining" in the sense of Foias and Temam [21]. 
Corollary 9. Let the hypotheses be those of the main theorem, and assume that $\mathcal{A}$ attracts in the $\mathbb{L}^{\infty}(\Omega)$ norm. Then almost every set of $k$ nodes is determining, provided that $k>16 n d_{f}(\mathcal{A})$.

Proof. We take a set of nodes which are instantaneously determining, as guaranteed by Theorem 1 , and consider $\mathcal{A}$ as a subset of $\mathbb{L}^{\infty}$. Note that Lemma 4 shows that the identity map on the attractor is continuous from $\mathbb{L}^{2}$ into $G_{\varphi \tau}$, and so certainly continuous from $\mathbb{L}^{2}$ into $\mathbb{L}^{\infty}$; it follows that $\mathcal{A}$ is a compact subset of $\mathbb{L}^{\infty}$.

Observe that the map from $\mathcal{A}$ into $\mathbb{R}^{k}$ given by

$$
u \mapsto E_{\mathbf{x}}(u)=\left(u\left(x_{1}\right), \ldots, u\left(x_{k}\right)\right)
$$

is continuous. Since it is also injective on $\mathcal{A}$ and $\mathcal{A}$ is compact, it follows that the map from $E_{\mathbf{x}}(\mathcal{A})$ into $\mathcal{A}$ given by $E_{\mathbf{x}}^{-1}$ is continuous. This implies that given an $\epsilon>0$ there exists a $\delta$ which can be taken $\leq \epsilon$ such that, if $u, v \in \mathcal{A}$ and

$$
\sup _{j}\left|u\left(x_{j}\right)-v\left(x_{j}\right)\right| \leq \delta,
$$

then

$$
\|u-v\|_{\infty} \leq \frac{1}{3} \epsilon
$$

Now suppose that $u(x, t)$ and $v(x, t)$ are two solutions which agree asymptotically on the set of nodes, as in (3). Since $\mathcal{A}$ is the attractor, given $\epsilon>0$ there exists a time $T$ such that

$$
\operatorname{dist}_{L^{\infty}}(u(x, t), \mathcal{A}) \leq \frac{1}{3} \delta, \quad \operatorname{dist}_{L^{\infty}}(v(x, t), \mathcal{A}) \leq \frac{1}{3} \delta,
$$

and

$$
\sup _{j}\left|u\left(x_{j}, t\right)-v\left(x_{j}, t\right)\right| \leq \frac{1}{3} \delta,
$$

for all $t \geq T$. It follows that there are functions $u^{*}(t)$ and $v^{*}(t)$ lying in $\mathcal{A}$ such that

$$
\left\|u^{*}(t)-u(t)\right\|_{\infty} \leq \frac{1}{3} \delta, \quad\left\|v^{*}(t)-v(t)\right\|_{\infty} \leq \frac{1}{3} \delta
$$

and

$$
\left|u^{*}\left(x_{j}, t\right)-v^{*}\left(x_{j}, t\right)\right| \leq \delta \quad \text { for all } 1 \leq j \leq k,
$$

and therefore $\left\|u^{*}(t)-v^{*}(t)\right\|_{\infty} \leq \frac{1}{3} \epsilon$. Combining this with (21) shows that

$$
\|u(t)-v(t)\|_{\infty} \leq \epsilon
$$

for all $t \geq T$, and we are done.

We note that this result gives estimates for the number of determining nodes in line with the dimension estimates of the attractor. For the 2D Navier-Stokes equations our $G^{2 / 3}(1+\log G)^{1 / 3}$ improves on the previous best estimate for the number of nodes was of the order of $G$ [36]. (Note, however, that this coarser bound is valid without the assumption of Gevrey regular forcing.)

However, it is worth pointing out that in 1D problems the analyticity of solutions can be exploited in a much stronger way - for example, Kukavica [38] has proved that for the 1D Ginzburg-Landau equation two nodes are asymptotically determining if they are close enough together; Oliver and Titi [49] do the same for weakly damped 
driven nonlinear Schrödinger equation, and Collet and Titi [6] for the CGLE on the whole real line. Foias and Kukavica [18] show that for the 1D Kuramoto-Sivashinsky, four nodal values suffice. Nonetheless, these results do not provide the instantaneously determining nodes of our Theorem 1.

\subsection{Other embedding results}

It is interesting to compare this result to the usual experimental method of reconstructing dynamics using Takens' time-delay embedding theorem [56]. Rather than taking measurements scattered throughout the spatial domain, this method consists of taking a series of measurements of some "observation function" $h$ at equally spaced time intervals.

This theorem has only been proved in the finite-dimensional case, and we give a statement valid for Lipschitz continuous differential equations on $\mathbb{R}^{n}$. In the formulation of Hunt and Kaloshin [33] (see also [55]), if the attractor has dimension $d$ then for a prevalent set of Lipschitz functions $h: \mathbb{R}^{n} \rightarrow \mathbb{R}$ and all $T$ sufficiently small, the map

$$
u \mapsto H[u]=(h[u(0)], h[u(T)], h[u(2 T)], \ldots, h[u(2 d T)])
$$

is $1-1$ on the attractor.

In this method each additional measurement recovers dynamical information, since once $h[u(0)], \ldots, h[u(2 d T)]$ are known, only $h[u((2 d+1) T]$ is required to determine $u(T)$, since

$$
H[u(T)]=(h[u(T)], h[u(2 T)], h[u(3 T)], \ldots, h[u((2 d+1) T)]) .
$$

The instantaneously determining nodes are much less efficient in this regard, since the only way to determine $u(T)$ is to take measurements at every node again at time $T$.

A version of the proof in this paper allows one to give, in certain circumstances, a related result which combines the delay-embedding approach with the distributed nodes approach, to show that a sufficient number of measurements, distributed in both space and time would be enough to determine a unique element on the attractor (cf. [55, Remark 2.9] in the finite-dimensional case). This is discussed in more detail in [52].

\section{Acknowledgements}

This work was undertaken while PKF was visiting Warwick over Easter 1999 — he would like to thank the Mathematics Institute for their financial support for this visit. JCR would like to thank José Langa for many interesting and stimulating conversations on this subject while he was visiting Oxford in the Spring of 1998, Peter Thomas, Greg King, and other members of the Fluid Dynamics Research Centre at Warwick for a morning of lively discussion, and Edriss Titi for his helpful emails.

\section{References}

[1] M. Bartucelli, P. Constantin, C. Doering, J.D. Gibbon, M. Gisselfält, On the possibility of soft and hard turbulence in the complex Ginzburg-Landau equation, Physica D 44 (1990) 421-444.

[2] C. Cao, M.A. Rammaha, E.S. Titi, Gevrey regularity for nonlinear analytic parabolic equations on the sphere, J. Dyn. Diff. Eq. 12 (2000) 411-433.

[3] B. Cockburn, D.A. Jones, E.S. Titi, Estimating the number of asymptotic degrees of freedom for nonlinear dissipative systems, Math. Comput. 66 (1997) 1073-1087.

[4] P. Collet, J.-P. Eckmann, H. Epstein, J. Stubbe, A global attracting set for the Kuramoto-Sivashinsky equation, Commun. Math. Phys. 152 (1993) 203-214. 
[5] P. Collet, J.-P. Eckmann, H. Epstein, J. Stubbe, Analyticity for the Kuramoto-Sivashinsky equation, Physica D 67 (1993) 321-326.

[6] P. Collet, E.S. Titi, Determining nodes for extended dissipative systems, Nonlinearity 9 (1996) 1089-1097.

[7] P. Constantin, C. Foias, R. Temam, Attractors representing turbulent flows, Mem. Am. Math. Soc. 53 (1985).

[8] P. Constantin, C. Foias, Navier-Stokes Equations, University of Chicago Press, Chicago, IL, 1988.

[9] P. Constantin, C. Foias, R. Temam, On the dimension of the attractors in two-dimensional turbulence, Physica D 30 (1988) $284-296$.

[10] A. Doelman, E.S. Titi, Regularity of solutions and the convergence of the Galerkin method in the Ginzburg-Landau equation, Numer. Func. Anal. Opt. 14 (1993) 299-321.

[11] C. Doering, J.D. Gibbon, D. Holm, B. Nicolaenko, Low-dimensional behaviour in the complex Ginzburg-Landau equation, Nonlinearity 1 (1988) 279-309.

[12] C. Doering, J.D. Gibbon, Applied Analysis of the Navier-Stokes Equations, Cambridge University Press, Cambridge, 1995.

[13] A. Eden, C. Foias, B. Nicolaenko, Z.S. She, Exponential attractors and their relevance to fluid dynamics systems, Physica D 63 (1993) $350-360$.

[14] A. Eden, C. Foias, B. Nicolaenko, R. Temam, Exponential Attractors for Dissipative Evolution Equations, RAM, Wiley, Chichester, UK, 1994.

[15] K. Falconer, The Geometry of Fractal Sets, Cambridge University Press, Cambridge, 1985.

[16] K. Falconer, Fractal Geometry, Wiley, Chichester, UK, 1990.

[17] A.B. Ferrari, E.S. Titi, Gevrey regularity for nonlinear analytic parabolic equations, Commun. Part. Diff. Eqs. 23 (1998) 1-16.

[18] C. Foias, I. Kukavica, Determining nodes for the Kuramoto-Sivashinksy equation, J. Dyn. Diff. Eq. 7 (1995) 365-373.

[19] C. Foias, O.P. Manley, R. Temam, Y. Treve, Asymptotic analysis of the Navier-Stokes equations, Physica D 9 (1983) 157-188.

[20] C. Foias, E.J. Olson, Finite fractal dimensions and Hölder-Lipschitz parametrization, Indiana Univ. Math. J. 45 (1996) $603-616$.

[21] C. Foias, R. Temam, Determination of the solutions of the Navier-Stokes equations by a set of nodal values, Math. Comput. 43 (1984) $117-133$.

[22] C. Foias, R. Temam, Gevrey class regularity for the solutions of the Navier-Stokes equations, J. Func. Anal. 87 (1989) $359-369$.

[23] C. Foias, E.S. Titi, Determining nodes, finite difference schemes and inertial manifolds, Nonlinearity 4 (1991) 135-153.

[24] P.K. Friz, J.C. Robinson, Smooth attractors have zero thickness, J. Math. Anal. Appl. 240 (1999) 37-46.

[25] P.K. Friz, I. Kukavica, J.C. Robinson, Nodal parametrisation of attractors for Dirichlet boundary conditions, Disr. Cont. Dyn. Syst., submitted for publication.

[26] J.D. Gibbon, E.S. Titi, Attractor dimension and small length scale estimates for the three-dimensional Navier-Stokes equations, Nonlinearity 10 (1997) 109-119.

[27] Z. Grujić, I. Kukavica, Space analyticity for the Navier-Stokes and related equations with initial data in $L^{p}$, J. Func. Anal. 152 (1998) $447-466$.

[28] Z. Grujić, I. Kukavica, Space analyticity for the nonlinear heat equation in a bounded domain, J. Diff. Eq. 154 (1999) $42-54$.

[29] Z. Grujić, Spatial analyticity on the global attractor for the Kuramoto-Sivashinsky equation, J. Dyn. Diff. Eq. 12 (2000) 217-228.

[30] J.K. Hale, Ordinary Differential Equations, Wiley, Chichester, UK, 1969.

[31] J.K. Hale, Asymptotic behaviour of dissipative systems, Mathematical Surveys and Monographs, Vol. 25, American Mathematical Society, Providence, RI, 1988.

[32] B.R. Hunt, T. Sauer, J.A. Yorke, Prevalence: a translation-invariant almost every for infinite dimensional spaces, Bull. Am. Math. Soc. 27 (1992) 217-238.

[33] B.R. Hunt, V.Y. Kaloshin, Regularity of embeddings of infinite-dimensional fractal sets into finite-dimensional spaces, Nonlinearity 12 (1999) 1263-1275.

[34] F. John, Partial Differential Equations, 4th Edition, Springer, New York, 1982.

[35] D.A. Jones, E.S. Titi, On the number of determining nodes for the 2D Navier-Stokes equations, Physica D 60 (1992) 72-88.

[36] D.A. Jones, E.S. Titi, Upper bounds on the number of determining modes, nodes, and volume elements for the Navier-Stokes equations, Indiana Univ. Math. J. 42 (1993) 1-12.

[37] R.H. Kraichnan, Inertial ranges in two-dimensional turbulence, Phys. Fluids 10 (1967) 1417-1423.

[38] I. Kukavica, On the number of determining nodes for the Ginzburg-Landau equation, Nonlinearity 5 (1992) 997-1006.

[39] I. Kukavica, An upper bound for the winding number for solutions of the Ginzburg-Landau equation, Indiana Univ. Math. J. 41 (1992) $825-836$.

[40] I. Kukavica, Oscillations of solutions of the Kuramoto-Sivashinksy equation, Physica D 76 (1994) 369-374.

[41] I. Kukavica, Hausdorff length of level sets for solutions of the Ginzburg-Landau equation, Nonlinearity 8 (1995) 113-129.

[42] C. Kuratowski, Topology, Vol. I, Academic Press, London, 1968.

[43] X. Liu, Gevrey class regularity and approximate inertial manifolds for the Kuramoto-Sivashinsky equation, Physica D 50 (1991) 135-151.

[44] H.V. Ly, E.S. Titi, Global Gevrey regularity for the Bénard convection in porous medium with zero Darcy-Prandtl number, J. Nonlinear Sci. 9 (1999) 333-362.

[45] R. Mañé, On the dimension of the compact invariant sets of certain nonlinear maps, Springer Lecture Notes in Mathematics, Vol. 898, Springer, Berlin, 1981, pp. 230-242.

[46] M. Marion, Attractors for reaction-diffusion equations: existence and estimate of their dimension, Appl. Anal. 25 (1987) $101-147$.

[47] M. Marion, Approximate inertial manifolds for reaction-diffusion equations in high space dimension, J. Dyn. Diff. Eq. 1 (1989) $245-267$. 
[48] B. Nicolaenko, B. Scheurer, R. Temam, Some global properties of the Kuramoto Sivashinsky equation: nonlinear stability and attractors, Physica D 16 (1985) 155-183.

[49] M. Oliver, E.S. Titi, Analyticity of the attractor and the number of determining nodes for a weakly damped driven nonlinear Schödinger equation, Indiana Univ. Math. J. 47 (1998) 49-73.

[50] K.S. Promislow, Time analyticity and Gevrey class regularity for solutions of a class of dissipative partial differential equations, Nonlinear Anal.: Theor. Meth. Appl. 16 (1991) 959-980.

[51] R.M. Range, Holomorphic Functions and Integral Representations in Several Complex Variables, Springer, New York, 1986.

[52] J.C. Robinson, A rigorous treatment of experimental observations for the two-dimensional Navier-Stokes equations, Proc. R. Soc. London A, in press.

[53] W. Rudin, Principles of Mathematical Analysis, McGraw-Hill, New York, 1976.

[54] W. Rudin, Real and Complex Analysis, McGraw-Hill, New York, 1974.

[55] T. Sauer, J.A. Yorke, M. Casdagli, Embedology, J. Stat. Phys. 71 (1993) 529-547.

[56] F. Takens, Detecting strange attractors in turbulence, Springer Lecture Notes in Mathematics 898 (1981) 366-381.

[57] R. Temam, Navier-Stokes Equations, North-Holland, Amsterdam, 1984.

[58] R. Temam, Infinite Dimensional Dynamical Systems in Mechanics and Physics, Vol. 68, Springer, Amsterdam, 1988.

[59] R. Temam, Navier-Stokes equations and nonlinear functional analysis, CBMS-NSF Regional Conference Series in Applied Mathematics, SIAM, Philadelphia, PA, 1983.

[60] M. Yamazato, Absolute continuity of operator-self-decomposable distributions on $\mathbb{R}^{d}$, J. Multivariate Anal. 13 (1983) 550-560.

[61] B.R. Hunt, T. Sauer, J.A. Yorke, Prevalence: an addendum, Bull. Am. Math. Soc. 28 (1993) 306-307. 